\title{
Association of thrombocytopenia and infection in patients with ST-elevation myocardial infarction undergoing percutaneous coronary intervention
}

Litao Wang ${ }^{1,2 \dagger}$, Weijiang $\mathrm{Su}^{3 \dagger}{ }^{\dagger}$, Jinhua Xue ${ }^{4 \dagger}$, Xiao Gong ${ }^{5}$, Yining Dai ${ }^{1}$, Jiyan Chen ${ }^{1}$, Ling Xue ${ }^{1}$, Pengcheng He ${ }^{1,2,6}$, Yuanhui Liu ${ }^{1^{*}}$ and Ning $\operatorname{Tan}^{1,2,6^{*}}$ (D)

\begin{abstract}
Background: The impact of thrombocytopenia on infection in patients with ST-elevation myocardial infarction (STEMI) remains poorly understood.

Aims: To evaluate the association between thrombocytopenia and infection in patients with STEMI.

Methods: Patients diagnosed with STEMI were identified from January 2010 to June 2016. The primary endpoint was in-hospital infection, and major adverse clinical events (MACE) and all-cause death were considered as secondary endpoints.

Results: A total of 1401 STEMI patients were enrolled and divided into two groups according to the presence ( $n=186)$ or absence $(n=1215)$ of thrombocytopenia. The prevalence of in-hospital infection was significantly higher in the thrombocytopenic group (30.6\% (57/186) vs. 16.2\% (197/1215), $p<0.001)$. Prevalence of in-hospital MACE (30.1\% (56/186) vs. 16.4\% (199/1215), $p<0.001)$ and all-cause death (8.1\% (15/186) vs. 3.8\% $(46 / 1215), p=0.008)$ revealed an increasing trend. Multivariate analysis indicated that thrombocytopenia was independently associated with increased in-hospital infection $(\mathrm{OR}, 2.09 ; 95 \% \mathrm{Cl} 1.32-3.27 ; p=0.001)$ and MACE $(1.92 ; 1.27-2.87 ; p=0.002)$, but not all-cause death $(1.87 ; 0.88-3.78 ; p=0.091)$. After a median follow-up of 2.85 years, thrombocytopenia was not associated with all-cause death at multivariable analysis (adjusted hazard ratio, 1.19; 95\% Cl 0.80-1.77; $p=0.383$ ).
\end{abstract}

Conclusions: Thrombocytopenia is significantly correlated with in-hospital infection and MACE, and might be used as a prognostic tool in patients with STEMI.

Keywords: Thrombocytopenia, Infection, ST-elevation myocardial infarction, Percutaneous coronary intervention

*Correspondence: liuyuanhui@gdph.org.cn; gdtanning@126.com

†Litao Wang, Weijiang Su and Jinhua Xue have contributed equally to this work

1 Department of Cardiology, Guangdong Cardiovascular Institute, Guangdong Provincial Key Laboratory of Coronary Heart Disease Prevention, Guangdong Provincial People's Hospital, Guangdong Academy of Medical Sciences, Guangzhou 510100, China

${ }^{2}$ Guangdong Provincial People's Hospital, School of Medicine, South

China University of Technology, Guangzhou 510100, China

Full list of author information is available at the end of the article

\section{Background}

Infection is an uncommon (reported prevalence of 2.4\%) but potentially devastating complication in patients with ST-elevation myocardial infarction (STEMI). Infection in these patients is usually associated with a significantly increased risk of mortality and morbidity, prolongs hospitalization, and increases healthcare costs [1-3]. Given most infections are considered preventable, there is an urgent need to identify STEMI patients at high risk 
of infection, and implement interventions to prevent infection.

Thrombocytopenia is a common laboratory abnormality in patients presenting with acute myocardial infarction (AMI) and always defined as a platelet count $<150 \times 10^{9} / \mathrm{L}$ [4]. Baseline thrombocytopenia showed strong predictive capacity for major adverse clinical events (MACE), ischemic target lesion revascularization and 1-year death among patients with acute coronary syndrome (ACS) undergoing percutaneous coronary intervention (PCI) [5]. Additionally, thrombocytopenia has been previously considered as a risk factor for worse outcomes and has been reported to be independently associated with in-hospital mortality in ACS patients [6]. However, few studies have explored the prognostic value of thrombocytopenia for infection in patients with STEMI. In terms of etiological factors, in addition to congenital thrombocytopenia and pseudothrombocytopenia, different acquired causes can be included. Of which, infectious agents play a crucial role for their contribution to a reduction in the platelet count by suppressing the bone marrow directly or increasing peripheral consumption of platelets [7-9].

Recent studies have reported that patients with coronavirus disease 2019 (COVID-19) exhibited severe thrombocytopenia [10], and Helicobacter pylori (H. pylori) infection was on the rise as a cause of immune thrombocytopenia (ITP) [11], while another research demonstrated that low platelet count was independently correlated with an increased risk of infection in patients with ITP [12], which indicated that infections were closely associated with thrombocytopenia. Furthermore, thrombocytopenia has been shown to be a poor prognostic risk factor for systemic infections among patients from medical, surgical, mixed, or trauma ICUs [13], and moderate thrombocytopenia (platelet count $<100 \times 10^{9} / \mathrm{L}$ ) was a potential risk stratification tool for severe Clostridium difficile infection (CDI) [14].

Given the potential value of thrombocytopenia in the prediction of infection, and in order to improve risk stratification for these patients, we sought to investigate the association between thrombocytopenia and infection in patients with STEMI.

\section{Methods}

\section{Study population}

Patients diagnosed with STEMI were enrolled from January 2010 to June 2016 in Guangdong Provincial People's Hospital. STEMI was diagnosed if patients presented within $12 \mathrm{~h}$ from the onset of symptoms including typical chest pain lasting for $\geq 30 \mathrm{~min}$, not responsive to nitrates, with ST-segment elevation of $\geq 0.2 \mathrm{mV}$ in at least two contiguous leads, or left bundle-branch block
[15]. The exclusion criteria were as follows: (a) with chronic inflammatory disease; (b) with chronic renal failure necessitating hemodialysis upon hospital admission; (c) undergoing cardiac surgery; (d) who died within $24 \mathrm{~h}$ after hospital admission; (e) without performing PCI; (f) without platelet count data and (g) receiving dual antiplatelet therapy (DAPT) or other medications potentially causing thrombocytopenia before admission. The study protocol was approved by Ethics Committee of our hospital. The study was carried out according to the Principles of the Declaration of Helsinki 1975 and its later amendments. Written informed consents were obtained from patients or their relatives.

\section{Study endpoints and follow-up}

Thrombocytopenia was defined as a platelet count $<150 \times 10^{9} / \mathrm{L}$ [4]. The primary endpoint was the development of infection during hospitalization, which was defined as infection requiring antibiotics (reflecting the clinical influence of infection compatible with the necessity for additional treatment) [16], and was determined in medical records in accordance with ICD-10-CM codes, including pneumonia (J18.9), pyelonephritis (N10), ureteritis (N28.86), urethritis (N34.1), cystitis (N30.90), and other infections (such as sepsis, A41.9; bacteremia, R78.81 or unspecified infectious disease, B99.9). Additionally, the types of infections included pulmonary, urinary, or other (including abdominal sepsis, primary bacteremia, and unidentified primary infection site) infections. The secondary endpoint was in-hospital MACE, including all-cause death, stroke, and any bleeding during hospitalization. Other endpoints were also documented, including all-cause death during hospitalization and follow-up. Patients were followed up through telephone-tracking methods or outpatient clinic interviews for $\geq 1$ year by trained nurses.

\section{Statistical analyses}

Continuous variables with a normal distribution were presented as mean $\pm S D$, and compared using two-sample $t$-tests. Continuous variables with a non-normal distribution were expressed as median and interquartile ranges, and analyzed using Wilcoxon rank sum tests. Categorical variables (which are presented as percentages) were compared using the chi-squared test or Fisher's exact test (as appropriate). In-hospital outcomes and follow-up outcomes were calculated using a logistic regression model or Cox regression model in multivariable analysis. As for sample size consideration, we applied a rule of thumb, namely, that the number of events per variable should be 10 or greater. We expected about no more than 11 factors in the development model; thus, at least 110 infections were needed. According to the 
incidence of infections (10\%) in the previous study [17], the final sample size of at least 1100 patients was needed. All tests were two-tailed, and $p<0.05$ was considered significant. Data analyses were carried out using SAS v9.4 (SAS Institute, Cary, NC, USA).

\section{Results}

A total of 2283 patients with STEMI at our hospital were identified between January 2010 and June 2016; of these, 882 patients were excluded according to the exclusion criteria. Finally, 1401 patients $(61.77 \pm 12.37$ years, 82.7\% male) diagnosed with STEMI undergoing PCI were enrolled and analyzed in this study (Fig. 1). During hospitalization, 186 (13.3\%) STEMI patients developed thrombocytopenia (platelet count $<150 \times 10^{9} / \mathrm{L}$ ). The clinical characteristics of the study population at baseline are shown in Table 1. Patients with thrombocytopenia were older and more likely to be men, than those not suffering from thrombocytopenia. A higher serum level of creatinine, and lower levels of total cholesterol and hemoglobin, were found in thrombocytopenic patients. Furthermore, thrombocytopenic patients had a higher prevalence of myocardial infarction and new use of antibiotics compared with non-thrombocytopenia cases. However, left ventricular ejection fraction (LVEF), procedural characteristics, and the prevalence of hypertension, diabetes mellitus, chronic obstructive pulmonary disease (COPD), and hyperlipemia were not significantly different between the two groups.
As shown in Table 2, thrombocytopenic patients were more likely to develop infection $(30.6 \%(57 / 186)$ vs. $16.2 \%(197 / 1215), p<0.001)$, with no significant differences in various types of infection $(p=0.804)$, as well as in-hospital MACE (30.1\% (56/186) vs. 16.4\% (199/1215), $p<0.001)$ than non-thrombocytopenia cases during hospitalization. The thrombocytopenia group also had a higher proportion of in-hospital death $(8.1 \%(15 / 186)$ vs. $3.8 \%(46 / 1215), p=0.008)$ and longer hospital stay $(11.41 \pm 10.17$ vs. $8.43 \pm 6.80$ days, $p<0.001)$ than the non-thrombocytopenia group (Table 2). Additionally, subgroup analysis revealed that $50 \leq$ PLT $<150$ was prominently associated with an increased risk of infection (odds ratio (OR), 2.21; 95\% confidence interval (CI) $1.55-3.12 ; p<0.001)$, but not severe thrombocytopenia defined as PLT $<50$ (OR, 5.02; 95\% CI 0.20-127.28; $p=0.255$ ) due to the small sample size possibly (Additional file 1: Table S1). Conversely, an obvious negative correlation between normal platelet count and infection was discovered (OR, 0.20; 95\% CI $0.17-0.23 ; p<0.001$ ) (Additional file 1: Table S1).

Univariate analysis suggested that thrombocytopenia was significantly associated with an elevated risk of infection (OR, 2.28; 95\%CI 1.61-3.22; $p<0.001)$. After adjustment for potential confounding factors (including age, Killip classification, diabetes, MI, stroke, serum albumin, WBC, hemoglobin, serum creatinine and LVEF), multivariate analysis showed that thrombocytopenia remained a significant predictor of infection risk in such patients

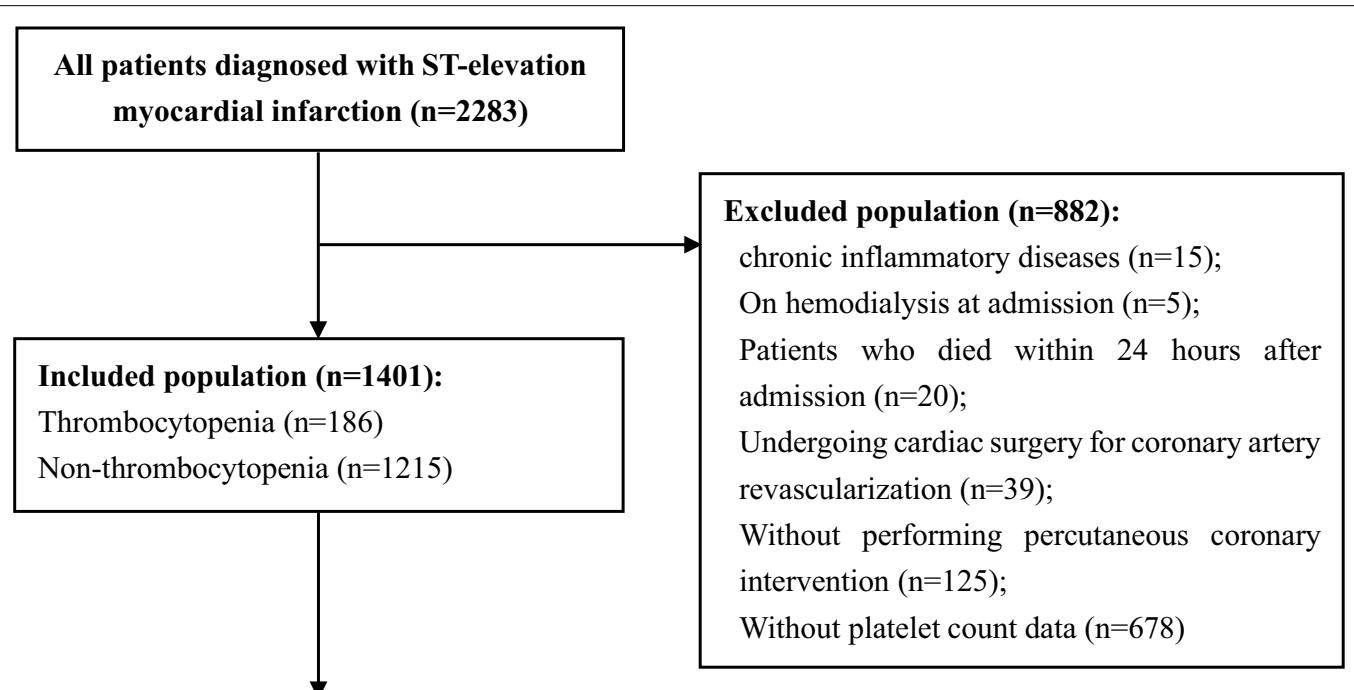

Primary endpoint: In-hospital infection Secondary endpoints: Major adverse clinical events; all-cause death

Fig. 1 Flowchart showing patient selection 
Table 1 Clinical characteristics of the study cohort

\begin{tabular}{|c|c|c|c|c|}
\hline Variables & Overall $(n=1401)$ & $\begin{array}{l}\text { Patients with } \\
\text { thrombocytopenia }(n=186)\end{array}$ & $\begin{array}{l}\text { Patients without } \\
\text { thrombocytopenia }(n=1215)\end{array}$ & $p$ value \\
\hline Platelet counts $\left(\times 10^{9} / \mathrm{L}\right)$ & $214.47 \pm 64.55$ & $125.81 \pm 23.01$ & $228.04 \pm 57.76$ & $<0.001$ \\
\hline Age (years) & $61.77 \pm 12.37$ & $65.99 \pm 11.06$ & $61.13 \pm 12.43$ & $<0.001$ \\
\hline Male, n (\%) & $1158(82.7 \%)$ & $157(84.4 \%)$ & $1001(82.4 \%)$ & 0.498 \\
\hline Female, n (\%) & $243(17.3 \%)$ & $29(15.6 \%)$ & $214(17.6 \%)$ & \\
\hline $\mathrm{SBP}(\mathrm{mmHg})$ & $122.00 \pm 21.64$ & $118.70 \pm 21.82$ & $122.50 \pm 21.58$ & 0.026 \\
\hline $\mathrm{DBP}(\mathrm{mmHg})$ & $73.48 \pm 12.76$ & $71.63 \pm 11.26$ & $73.77 \pm 12.96$ & 0.034 \\
\hline Heart rate (bpm) & $80.71 \pm 16.54$ & $80.78 \pm 18.98$ & $80.70 \pm 16.14$ & 0.954 \\
\hline Killip classification & $1.42 \pm 0.77$ & $1.48 \pm 0.88$ & $1.41 \pm 0.75$ & 0.236 \\
\hline Hypertension, n (\%) & $724(51.7 \%)$ & $97(52.2 \%)$ & $627(51.6 \%)$ & 0.890 \\
\hline Diabetes, n (\%) & $367(26.2 \%)$ & $54(29.0 \%)$ & $313(25.8 \%)$ & 0.345 \\
\hline Hyperlipemia, n (\%) & $145(10.3 \%)$ & $14(7.5 \%)$ & $131(10.8 \%)$ & 0.175 \\
\hline Smoke, n (\%) & $622(44.4 \%)$ & $83(44.6 \%)$ & $549(44.4 \%)$ & 0.947 \\
\hline COPD, n (\%) & $34(2.4 \%)$ & $6(3.2 \%)$ & $28(2.3 \%)$ & 0.447 \\
\hline Prior MI, n (\%) & $72(5.1 \%)$ & $16(8.6 \%)$ & $56(4.6 \%)$ & 0.022 \\
\hline Prior stroke, n (\%) & $96(6.9 \%)$ & $19(10.2 \%)$ & $77(6.3 \%)$ & 0.051 \\
\hline Bleeding, n (\%) & $221(15.8 \%)$ & $46(24.7 \%)$ & $175(14.4 \%)$ & $<0.001$ \\
\hline Atrial fibrillation, n (\%) & $44(3.1 \%)$ & $76(3.8 \%)$ & $37(3.0 \%)$ & 0.601 \\
\hline CABG, n (\%) & $3(0.2 \%)$ & $0(0.0 \%)$ & $3(0.2 \%)$ & 0.498 \\
\hline Prior PCl, n (\%) & $140(10.0 \%)$ & $16(8.6 \%)$ & $124(10.2 \%)$ & 0.497 \\
\hline \multicolumn{5}{|l|}{ Laboratory characteristics } \\
\hline White blood cell $\left(\times 10^{9} / \mathrm{L}\right)$ & $11.82 \pm 3.83$ & $10.93 \pm 3.52$ & $11.95 \pm 3.86$ & $<0.001$ \\
\hline Serum creatinine $(\mu \mathrm{mol} / \mathrm{L})$ & $103.78 \pm 74.70$ & $124.43 \pm 104.83$ & $100.61 \pm 68.44$ & $<0.001$ \\
\hline Triglycerides (mmol/L) & $1.57 \pm 1.20$ & $1.35 \pm 1.55$ & $1.60 \pm 1.14$ & 0.010 \\
\hline Total cholesterol (mmol/L) & $4.83 \pm 1.19$ & $4.33 \pm 1.06$ & $4.91 \pm 1.19$ & $<0.001$ \\
\hline LDL-C (mmol/L) & $3.07 \pm 1.01$ & $2.65 \pm 0.86$ & $3.14 \pm 1.02$ & $<0.001$ \\
\hline $\mathrm{HbA1c}(\%)$ & $6.70 \pm 1.73$ & $6.85 \pm 1.70$ & $6.68 \pm 1.74$ & 0.271 \\
\hline Serum albumin (g/L) & $33.26 \pm 4.29$ & $32.41 \pm 4.11$ & $33.39 \pm 4.31$ & 0.004 \\
\hline Hemoglobin (g/L) & $136.24 \pm 18.31$ & $132.03 \pm 19.59$ & $136.88 \pm 18.03$ & $<0.001$ \\
\hline LVEF (\%) & $52.00 \pm 11.71$ & $50.79 \pm 12.24$ & $52.19 \pm 11.61$ & 0.130 \\
\hline \multicolumn{5}{|l|}{ Medication during hospital stay } \\
\hline Statins, n (\%) & 1384 (98.8\%) & $178(95.7 \%)$ & $1206(99.3 \%)$ & $<0.001$ \\
\hline Aspirin, n (\%) & 1370 (97.8\%) & $181(97.3 \%)$ & 1189 (97.9\%) & 0.636 \\
\hline Clopidogrel, n (\%) & 1384 (98.8\%) & $178(95.7 \%)$ & $1206(99.3 \%)$ & 0.593 \\
\hline CCB, n (\%) & $149(10.6 \%)$ & $27(14.5 \%)$ & $122(10.0 \%)$ & 0.065 \\
\hline Olmesartan, n (\%) & $225(16.1 \%)$ & $30(16.1 \%)$ & $195(16.0 \%)$ & 0.978 \\
\hline New antibiotic use, n (\%) & $240(17.1 \%)$ & $53(28.5 \%)$ & $187(15.4 \%)$ & $<0.001$ \\
\hline \multicolumn{5}{|l|}{ Procedural characteristics } \\
\hline Radial access, n (\%) & $1191(85.0 \%)$ & 155 (83.3\%) & $1036(85.3 \%)$ & 0.491 \\
\hline Femoral assess, n (\%) & $210(15.0 \%)$ & $31(16.7 \%)$ & $179(14.7 \%)$ & \\
\hline Contrast volume, (mL) & $114.01 \pm 42.81$ & $117.86 \pm 42.40$ & $113.41 \pm 42.86$ & 0.213 \\
\hline Number of stents, (n) & $1.30 \pm 0.82$ & $1.38 \pm 0.88$ & $1.28 \pm 0.81$ & 0.153 \\
\hline Total length of stent, (mm) & $32.51 \pm 22.38$ & $34.67 \pm 23.04$ & $32.28 \pm 22.27$ & 0.158 \\
\hline
\end{tabular}

Data are presented as mean \pm SD or number (percentage)

$S B P$ systolic blood pressure, DBP diastolic blood pressure, COPD chronic obstructive pulmonary disease, $M I$ myocardial infarction, $T C$ total cholesterol, $C A B G$ coronary artery bypass grafting, $P C I$ percutaneous coronary intervention, $L D L-C$ low-density lipoprotein cholesterol, $L V E F$ left ventricular ejection fraction, $C C B$ calcium channel blockers 
Table 2 In-hospital adverse events of the study cohort

\begin{tabular}{|c|c|c|c|c|}
\hline Variables & Overall $(n=1401)$ & $\begin{array}{l}\text { Patients with } \\
\text { thrombocytopenia }(n=186)\end{array}$ & $\begin{array}{l}\text { Patients without } \\
\text { thrombocytopenia }(n=1215)\end{array}$ & $p$ value \\
\hline In-hospital infection, n (\%) & $254(18.1 \%)$ & $57(30.6 \%)$ & 197 (16.2\%) & $<0.001$ \\
\hline Type of infections, n (\%) & & & & 0.804 \\
\hline Pulmonary infection & 199 (78.3\%) & $43(75.4 \%)$ & $156(79.2 \%)$ & \\
\hline Urinary infection & $33(13.0 \%)$ & $8(14.0 \%)$ & $25(12.7 \%)$ & \\
\hline Other infections & $22(8.7 \%)$ & $6(10.5 \%)$ & $16(8.1 \%)$ & \\
\hline MACE, n (\%) & 255 (18.2\%) & $56(30.1 \%)$ & 199 (16.4\%) & $<0.001$ \\
\hline In-hospital death, n (\%) & $61(4.4 \%)$ & $15(8.1 \%)$ & $46(3.8 \%)$ & 0.008 \\
\hline Hospital day (d) & $8.82 \pm 7.40$ & $11.41 \pm 10.17$ & $8.43 \pm 6.80$ & $<0.001$ \\
\hline
\end{tabular}

Data are presented as mean $\pm S D$ or number (percentage)

MACE major adverse clinical events

Table 3 Adjusted OR and 95\% Cl of thrombocytopenia for infection, MACE and all-cause death at multivariate analysis

\begin{tabular}{llll}
\hline Variables & \multicolumn{4}{l}{ Thrombocytopenia } \\
\cline { 2 - 4 } & OR & $\mathbf{9 5 \% C l}$ & $\boldsymbol{p}$ value \\
\hline Infection & 2.09 & $1.32-3.27$ & 0.001 \\
MACE & 1.92 & $1.27-2.87$ & 0.002 \\
All-cause death & 1.87 & $0.88-3.78$ & 0.091 \\
\hline
\end{tabular}

Adjusted for age, Killip classification, diabetes, myocardial infarction, stroke, serum albumin, white blood cell, hemoglobin, serum creatinine and left ventricular ejection fraction

$O R$ odds ratio, $\mathrm{Cl}$ confidence interval, MACE major adverse clinical events

(OR, 2.09; 95\%CI 1.32-3.27; $p=0.001$ ) (Table 3). Similar results were observed for the prevalence of in-hospital MACE (OR, 1.92; 95\%CI 1.27-2.87; $p=0.002$ ) but not all-cause death (OR, 1.87; 95\%CI $0.88-3.78 ; p=0.091$ ) (Table 3).

After a median follow-up of 2.85 years, univariate Cox proportional hazard regression analyses showed that thrombocytopenia was independently associated with increased all-cause death (hazard ratio (HR), 1.52; 95\%CI $1.04-2.20 ; p=0.029$ ). However, upon fully adjusted multivariate Cox regression analysis, this significant association disappeared (adjusted HR, 1.19; 95\%CI 0.80-1.77; $p=0.383)$ (Table 4).

\section{Discussion}

The present study investigated, for the first time, the relationship between thrombocytopenia and infection in patients with STEMI undergoing PCI. We found that thrombocytopenia was associated with a significantly increased risk of in-hospital infection, in-hospital MACE but not all-cause death. These findings suggested that serial monitoring of platelet counts was essential to identify patients with STEMI at high risk of adverse outcomes.
Table 4 Cox proportional hazards regression analysis of factors associated with all-cause death

\begin{tabular}{lllr}
\hline Variables & HR & $\mathbf{9 5 \% C l}$ & $\boldsymbol{p}$ value \\
\hline Thrombocytopenia & 1.19 & $0.80-1.77$ & 0.383 \\
Age & 2.54 & $1.80-3.59$ & $<0.001$ \\
Killip classification & 1.51 & $1.29-1.77$ & $<0.001$ \\
Diabetes & 1.22 & $0.88-1.68$ & 0.235 \\
Myocardial infarction & 1.57 & $0.93-2.66$ & 0.094 \\
Stroke & 1.76 & $1.13-2.75$ & 0.013 \\
Serum albumin & 0.73 & $0.48-1.10$ & 0.128 \\
White blood cell & 1.56 & $1.10-2.20$ & 0.013 \\
Hemoglobin & 0.10 & $0.99-1.04$ & 0.311 \\
Serum creatinine & 1.02 & $1.01-1.04$ & $<0.001$ \\
LVEF & 0.39 & $0.28-0.55$ & $<0.001$ \\
\hline
\end{tabular}

$H R$ hazard ratio, $\mathrm{Cl}$ confidence interval, LEVF left ventricular ejection fraction

Platelets are anucleate blood cells that play a crucial role in the maintenance of hemostasis [18]. Thrombocytopenia has been evaluated in various cardiovascular procedures [19, 20], and been proven to be associated with worse short-term and long-term outcomes in patients with ACS [5]. Williamson and colleagues reported that critically ill patients with thrombocytopenia had an increased risk of death compared with patients with a normal platelet count [21]. An increased risk of death, ischemic events, and bleeding has also been reported in thrombocytopenic patients with ACS without ST elevation upon electrocardiography [22]. In patients suffering from sepsis, thrombocytopenia reflected proinflammatory stimuli and pro-hemostatic mechanisms [23], which suggested that the more the pronounced infectious stimulus, the greater the risk of thrombocytopenia. Additionally, as reported previously, acute and chronic infectious diseases were common causes of thrombocytopenia [24]. However, the prognostic value of thrombocytopenia for infection remains unclear. 
In our analyses, we demonstrated that the prevalence of thrombocytopenia in STEMI patients was 13.3\%, while previous studies have reported the incidence of thrombocytopenia in patients with ACS varied from 1.6 to $13 \%$ $[25,26]$. The mechanisms of platelet loss in such patients have not been illustrated explicitly because patients with thrombocytopenia are frequently excluded from large clinical trials [27]. However, anti-platelet agents and anticoagulation, especially heparin, are employed after surgical as well as conservative treatment, and may cause heparin-induced thrombocytopenia when antibodies react with complexes of heparin molecules and platelet factor-4 [28]. Furthermore, scholars have reported ischemic necrosis in patients presenting with myocardial dysfunction, was able to facilitate inflammation at the cellular level, and subsequently increased platelet clearance by macrophages as well as became the dominant stimulus for thrombocytopenia [26].

With regard to the predictive value of thrombocytopenia for infection and the underlying mechanisms of the association between them, some factors might be notable. First, patients with thrombocytopenia receive many transfusions of red blood cells and platelets in necessity, both of these interventions are proinflammatory [29, 30]. Second, infectious agents, with their prominent role in the causes of thrombocytopenia, help to decrease the platelet number by suppressing bone marrow directly or increasing peripheral consumption of platelets $[8$, 9]. Thus, to some extent, thrombocytopenia is able to reflect infection indirectly. Third, studies have demonstrated that platelets have a crucial role in response to infection by promoting translocation of immune cells to inflamed areas and releasing cytokines and other molecular mediators. Therefore, thrombocytopenia might inhibit platelet effects against infection by reducing the number of platelets and their function, which might be conducive to increasing the prevalence of in-hospital infection [31, 32]. Furthermore, platelets could inhibit macrophage-dependent inflammation, or enhance interleukin-10 secretion and reduce tumor necrosis factor- $\alpha$ $(\mathrm{TNF}-\alpha)$ secretion during infectious and noninfectious systemic inflammation [33, 34]. Finally, in gram-negative pneumonia-derived sepsis in mice, thrombocytopenia was associated with a strongly impaired host defense via enhancing proinflammatory cytokine release and bacterial growth, and disrupting vascular integrity and activating endothelial cell [35].

In addition to thrombocytopenia, our study firstly found that the levels of serum albumin and hemoglobin was independently associated with the risk of infection. Leonardi and colleagues reported that declining hemoglobin content, with or without overt bleeding, was independently associated with heightened risk of 1-year mortality among patients with ACS managed invasively [36]. Plakht et al. demonstrated that decreased serum albumin level on admission was an independent prognostic marker of increased long-term all-cause mortality in patients with AMI [37], while hypoalbuminemia was also found to be significantly associated with worse in-hospital outcomes in patients with ACS [38]. However, we did not find that the levels of serum albumin and hemoglobin were associated with the risk of all-cause death, which might be attributed to the little sample size and low incidence of all-cause death in our study.

Our study highlights the notion that thrombocytopenia is infrequent in patients diagnosed with STEMI, but is associated with a markedly increased prevalence of infection and in-hospital MACE, and should not be underestimated. Early identification of thrombocytopenia is essential for appropriate clinical care of STEMI patients. Hence, dynamic monitoring of platelet count might be encouraged for cardiologists among high-risk patients to avoid complications.

This study also had several main limitations. First, the causes of thrombocytopenia were not elucidated clearly, and the diagnosis of thrombocytopenia according to one complete blood count (CBC) test might be not sufficient while minor variation might occur and platelet agglutination might result in technical CBC diagnosis of thrombocytopenia. Second, our study was carried out at a single center with a relatively small study cohort. Third, we evaluated a cohort of Chinese patients, so caution is needed when trying to apply our conclusions to another population. Fourth, we failed to collect the information about blood product transfusion, which might be a potential confounding factor. Finally, because of a lack of studies investigating the outcome of infection in thrombocytopenia patients, the potential molecular mechanisms could not be explored.

\section{Conclusions}

In summary, the present study indicated that thrombocytopenia during hospitalization was independently associated with an increased prevalence of infection and in-hospital MACE in patients with STEMI. Thrombocytopenia could be used as a simple prognostic tool for these patients.

\section{Abbreviations}

STEMI: ST-elevation myocardial infarction; AMI: Acute myocardial infarction; ACS: Acute coronary syndrome; PCl: Percutaneous coronary intervention; MACE: Major adverse clinical events; ITP: Immune thrombocytopenia; DAPT: Dual antiplatelet therapy; TNF-a: Tumor necrosis factor-a; SBP: Systolic blood pressure; DBP: Diastolic blood pressure; COPD: Chronic obstructive pulmonary disease; TC: Total cholesterol; CABG: Coronary artery bypass grafting; LDL-C: Low-density lipoprotein cholesterol; LVEF: Left ventricular ejection fraction; CCB: Calcium channel blockers; CBC: Complete blood count. 


\section{Supplementary Information}

The online version contains supplementary material available at https://doi. org/10.1186/s12872-021-02210-3.

Additional file 1: Table S1. Association of platelet count on infection after multivariable adjustment.

\section{Acknowledgements}

The concise abstract was presented previously at the Great Wall International Congress of Cardiology 2020 (GW-ICC 2020) for academic communication.

\section{Authors' contributions}

Conception/Design: NT, YHL, PCH. Collection and/or assembly of data: LTW, YHL, WJS, JHX, YND. Data analysis and interpretation: YHL, LTW, WJS, JHX, XG, YND. Manuscript writing: LTW, YHL. Manuscript revising: NT, YHL, PCH, LX, JYC. Final approval of the version to be published: All authors. All authors read and approved the final manuscript.

\section{Funding}

This study was supported by a grant from the Shuangqing Talent Program Project of Guangdong Provincial people's Hospital (No. KJ012019095 and KJ012019084), the first clinical research training course "seed fund" of Guangdong Provincial people's Hospital (No.2018lcpx04), Bureau of Education of Jiangxi province (No. GJJ180814) and Key projects of Gannan Medical University (No. ZD201807). The funders had no role in the study design, data collection and analysis, the decision to publish, or the preparation of the manuscript. The work was not funded by any industry sponsors.

\section{Availability of data and materials}

The datasets used and/or analyzed in this study are available from the corresponding author on reasonable request.

\section{Declarations}

\section{Ethics approval and consent to participate}

This research was approved by the Ethics Committee of Guangdong Provincial People's Hospital (GDREC2016378H). The written informed consent was obtained from almost all of the patients before the procedure, and some informed consents were acquired from their next of kin for patients who could not sign informed consent themselves due to the chest pain or other reasonable causes.

\section{Consent for publication}

Not applicable.

\section{Competing interests}

The authors declare that they have no competing interests.

\begin{abstract}
Author details
${ }^{1}$ Department of Cardiology, Guangdong Cardiovascular Institute, Guangdong Provincial Key Laboratory of Coronary Heart Disease Prevention, Guangdong Provincial People's Hospital, Guangdong Academy of Medical Sciences, Guangzhou 510100, China. ${ }^{2}$ Guangdong Provincial People's Hospital, School of Medicine, South China University of Technology, Guangzhou 510100, China. ${ }^{3}$ Department of Cardiology, The People's Hospital of Dianbai District, Maoming 525400, China. ${ }^{4}$ Department of Physiology, School of Basic Medical Sciences, Key Laboratory of Prevention and Treatment of Cardiovascular and Cerebrovascular Diseases of Ministry of Education, Gannan Medical University, Ganzhou 341000, China. ${ }^{5}$ School of Public Health, Guangdong Pharmaceutical University, Guangzhou 510006, China. ${ }^{6}$ The Second School of Clinical Medicine, Southern Medical University, Guangzhou 510515, China.
\end{abstract}

Received: 11 October 2020 Accepted: 16 August 2021

Published online: 21 August 2021

\section{References}

1. Aggarwal B, Ellis SG, Lincoff AM, Kapadia SR, Cacchione J, Raymond RE, et al. Cause of death within 30 days of percutaneous coronary intervention in an era of mandatory outcome reporting. J Am Coll Cardiol. 2013:62(5):409-15.

2. Pedersen F, Butrymovich V, Kelbæk H, Wachtell K, Helqvist S, Kastrup $J$, et al. Short- and long-term cause of death in patients treated with primary PCI for STEMI. J Am Coll Cardiol. 2014;64(20):2101-8.

3. Truffa AA, Granger CB, White KR, Newby LK, Mehta RH, Hochman JS, et al. Serious infection after acute myocardial infarction: incidence, clinical features, and outcomes. JACC Cardiovasc Interv. 2012;5(7):769-76.

4. Cheng CK, Chan J, Cembrowski GS, van Assendelft OW. Complete blood count reference interval diagrams derived from NHANES III: stratification by age, sex, and race. Lab Hematol. 2004;10(1):42-53.

5. Yadav M, Généreux P, Giustino G, Madhavan MV, Brener SJ, Mintz G, et al. Effect of baseline thrombocytopenia on ischemic outcomes in patients with acute coronary syndromes who undergo percutaneous coronary intervention. Can J Cardiol. 2016:32(2):226-33.

6. Overgaard CB, Ivanov J, Seidelin PH, Todorov M, Mackie K, Dzavík V. Thrombocytopenia at baseline is a predictor of inhospital mortality in patients undergoing percutaneous coronary intervention. Am Heart J. 2008;156(1):120-4.

7. Liebman HA. Viral-associated immune thrombocytopenic purpura. Hematol Am Soc Hematol Educ Program. 2008;2008:212-8.

8. Flaujac C, Boukour S, Cramer-Bordé E. Platelets and viruses: an ambivalent relationship. Cell Mol Life Sci. 2010;67(4):545-56.

9. Assinger A. Platelets and infection-an emerging role of platelets in viral infection. Front Immunol. 2014;5:649.

10. Dhama K, Patel SK, Pathak M, Yatoo MI, Tiwari R, Malik YS, et al. An update on SARS-CoV-2/COVID-19 with particular reference to its clinical pathology, pathogenesis, immunopathology and mitigation strategies. Travel Med Infect Dis. 2020;37:101755.

11. Lee A, Hong J, Chung H, Koh Y, Cho SJ, Byun JM, et al. Helicobacter pylori eradication affects platelet count recovery in immune thrombocytopenia. Sci Rep. 2020;10(1):9370.

12. Qu M, Liu Q, Zhao HG, Peng J, Ni H, Hou M, et al. Low platelet count as risk factor for infections in patients with primary immune thrombocytopenia: a retrospective evaluation. Ann Hematol. 2018;97(9):1701-6.

13. Hui P, Cook DJ, Lim W, Fraser GA, Arnold DM. The frequency and clinical significance of thrombocytopenia complicating critical illness: a systematic review. Chest. 2011;139(2):271-8.

14. Fountain EM, Moses MC, Park LP, Woods CW, Arepally GM. Thrombocytopenia in hospitalized patients with severe clostridium difficile infection. J Thromb Thrombolysis. 2017:43(1):38-42.

15. Ibanez B, James S, Agewall S, Antunes MJ, Bucciarelli-Ducci C, Bueno $H$, et al. 2017 ESC Guidelines for the management of acute myocardial infarction in patients presenting with ST-segment elevation: the task force for the management of acute myocardial infarction in patients presenting with ST-segment elevation of the European Society of Cardiology (ESC). Eur Heart J. 2018;39(2):119-77.

16. Feistritzer HJ, Kurz T, Stachel G, Hartung P, Lurz P, Eitel I, et al. General versus local anesthesia with conscious sedation in transcatheter aortic valve implantation: the randomized SOLVE-TAVI trial. Circulation. 2020;142(15):1437-47.

17. Putot A, Chague F, Manckoundia P, Cottin Y, Zeller M. Post-infectious myocardial infarction: new insights for improved screening. J Clin Med. 2019;8(6):827.

18. George JN. Platelets. Lancet. 2000;355:1531-9.

19. Ayoub K, Marji M, Ogunbayo G, Masri A, Abdel-Latif A, Ziada K, et al. Impact of chronic thrombocytopenia on in-hospital outcomes after percutaneous coronary intervention. JACC Cardiovasc Interv. 2018:11(18):1862-8.

20. Kertai MD, Zhou S, Karhausen JA, Cooter M, Jooste E, Li Y-J, et al. Platelet counts, acute kidney injury, and mortality after coronary artery bypass grafting surgery. Anesthesiology. 2016;124(2):339-52. 
21. Williamson DR, Lesur O, Tétrault JP, Nault V, Pilon D. Thrombocytopenia in the critically ill: prevalence, incidence, risk factors, and clinical outcomes. Can J Anaesth. 2013;60(7):641-51.

22. Eikelboom JW, Anand SS, Mehta SR, Weitz JI, Yi C, Yusuf S. Prognostic significance of thrombocytopenia during hirudin and heparin therapy in acute coronary syndrome without ST elevation: organization to assess strategies for ischemic syndromes (OASIS-2) study. Circulation. 2001;103(5):643-50.

23. Stéphan F, Hollande J, Richard O, Cheffi A, Maier-Redelsperger M, Flahault A. Thrombocytopenia in a surgical ICU. Chest. 1999;115(5):1363-70.

24. Fountain EM, Arepally GM. Etiology and complications of thrombocytopenia in hospitalized medical patients. J Thromb Thrombolysis. 2017:43(4):429-36.

25. Gore JM, Spencer FA, Gurfinkel EP, López-Sendón J, Steg PG, Granger CB, et al. Thrombocytopenia in patients with an acute coronary syndrome (from the global registry of acute coronary events [GRACE]). Am J Cardiol. 2009;103(2):175-80.

26. Wang TY, Ou FS, Roe MT, Harrington RA, Ohman EM, Gibler WB, et al. Incidence and prognostic significance of thrombocytopenia developed during acute coronary syndrome in contemporary clinical practice. Circulation. 2009;119(18):2454-62.

27. McCarthy CP, Steg G, Bhatt DL. The management of antiplatelet therapy in acute coronary syndrome patients with thrombocytopenia: a clinical conundrum. Eur Heart J. 2017;38(47):3488-92.

28. Modi C, Satani D, Cervellione KL, Cervantes J, Gintautas J. Delayed-onset heparin-induced thrombocytopenia type-2 during fondiparinux (Arixtra) therapy. Proc West Pharmacol Soc. 2009;52:5-7.

29. Twomley KM, Rao SV, Becker RC. Proinflammatory, immunomodulating, and prothrombotic properties of anemia and red blood cell transfusions. J Thromb Thrombolysis. 2006;21(2):167-74.

30. Stolla M, Refaai MA, Heal JM, Spinelli SL, Garraud O, Phipps RP, et al. Platelet transfusion - the new immunology of an old therapy. Front Immunol. 2015;6:28.
31. Ali RA, Wuescher LM, Worth RG. Platelets: essential components of the immune system. Curr Trends Immunol. 2015;16:65-78.

32. Morrell CN, Aggrey AA, Chapman LM, Modjeski KL. Emerging roles for platelets as immune and inflammatory cells. Blood. 2014;123(18):2759-67.

33. Xiang B, Zhang G, Guo L, Li XA, Morris AJ, Daugherty A, et al. Platelets protect from septic shock by inhibiting macrophage-dependent inflammation via the cyclooxygenase 1 signalling pathway. Nat Commun. 2013:4:2657.

34. Gudbrandsdottir S, Hasselbalch HC, Nielsen CH. Activated platelets enhance IL-10 secretion and reduce TNF-a secretion by monocytes. J Immunol. 2013;191(8):4059-67.

35. de Stoppelaar SF, van't Veer C, Claushuis TA, Albersen BJ, Roelofs JJ, van der Poll T. Thrombocytopenia impairs host defense in gram-negative pneumonia-derived sepsis in mice. Blood. 2014;124(25):3781-90.

36. Leonardi S, Gragnano F, Carrara G, Gargiulo G, Frigoli E, Vranckx P, et al. Prognostic implications of declining hemoglobin content in patients hospitalized with acute coronary syndromes. J Am Coll Cardiol. 2021;77(4):375-88.

37. Plakht $Y$, Gilutz $H$, Shiyovich A. Decreased admission serum albumin level is an independent predictor of long-term mortality in hospital survivors of acute myocardial infarction. Soroka Acute Myocardial Infarction II (SAMI-II) project. Int J Cardiol. 2016;219:20-4.

38. Hartopo AB, Gharini PP, Setianto BY. Low serum albumin levels and in-hospital adverse outcomes in acute coronary syndrome. Int Heart J. 2010;51(4):221-6.

\section{Publisher's Note}

Springer Nature remains neutral with regard to jurisdictional claims in published maps and institutional affiliations.
Ready to submit your research? Choose BMC and benefit from:

- fast, convenient online submission

- thorough peer review by experienced researchers in your field

- rapid publication on acceptance

- support for research data, including large and complex data types

- gold Open Access which fosters wider collaboration and increased citations

- maximum visibility for your research: over $100 \mathrm{M}$ website views per year

At $\mathrm{BMC}$, research is always in progress.

Learn more biomedcentral.com/submissions 